1 Title: ComEB protein is dispensable for transformation but must be translated for

2 optimal synthesis of ComEC

5 Authors: Micaela De Santis ${ }^{\mathrm{a}}$, Jeanette Hahn ${ }^{\mathrm{a}}$ and David Dubnau ${ }^{\text {a* }}$

7 Affiliation:

8 a Public Health Research Institute Center

9 New Jersey Medical School

10 Rutgers University

11 Newark, NJ, USA

\title{
13 *Correspondence
}

14 David Dubnau

15 Public Health Research Institute Center

16 New Jersey Medical School

17 Rutgers University

18 Newark, NJ, USA

19 dubnauda@njms.rutgers.edu

\section{Funding information}

22 National Institutes of General Medicine

23 Grant number R01GM057720

25 Keywords: transformation, translational coupling, nucleotide scavenging, ComEB,

26 ComEC, post transcriptional regulation

28 Data availability statement: The data that support the findings of this study are

29 available from the corresponding author upon reasonable request.

31 Running title: ComEB protein is not needed for transformation 


\section{Summary}

34 We show that the ComEB protein is not required for transformation in Bacillus

35 subtilis, despite its expression from within the comE operon under competence control.

36 We show further that the synthesis of the putative channel protein ComEC is

37 translationally coupled to the upstream comEB open reading frame, so that translation of

38 comEB and a suboptimal ribosomal binding site embedded in its sequence are needed for

39 proper comEC expression. Translational coupling appears to be a common mechanism in

40 three major competence operons for the adjustment of protein amounts independent of

41 transcriptional control, probably ensuring the correct stoichiometries for assembly of the

42 transformation machinery. comEB and comFC respectively encode cytidine deaminase

43 and a protein resembling type 1 phosphoribosyl transferases and we speculate that

44 nucleotide scavenging proteins are produced under competence control for efficient

45 reutilization of the products of degradation of the non-transforming strand during DNA

46 uptake. 


\section{Introduction}

Bacterial transformation is an important mode of horizontal gene transfer and a complete understanding of its mechanism requires the identification of all the genes and proteins required for the binding, uptake and integration of transforming DNA. Although most of the essential players have been identified (Blokesch, 2016, Dubnau \& Blokesch, 2019, Maier, 2020), uncertainty remains in the case of ComEB.

The conserved ComEA and ComEC proteins, are needed for transformation in nearly all bacteria, and at least in the firmicutes are encoded in the comE operon, which also encodes ComEB. ComEA is a DNA binding protein that participates in the uptake of transforming DNA to the periplasm (Provvedi \& Dubnau, 1999, Gangel et al., 2014,

59 Matthey \& Blokesch, 2016, Schwarzenlander et al., 2009), while ComEC is a large,

60 multipass membrane protein that most likely forms a channel for DNA transport to the

61 cytoplasm (Draskovic \& Dubnau, 2005). In Bacillus subtilis and in many other

62 firmicutes, the comE operon also encodes ComEB, a dCMP deaminase (Burghard-Schrod

63 et al., 2020, Oehlenschlaeger et al., 2015), while in other bacteria only comEA and

64 comEC are present. It has been suggested that ComEB is dispensable for the

65 transformation of B. subtilis, because a short in-frame deletion (comEB $\left.{ }^{484}\right)$, removing 28

66 out of 189 codons from the 5' end of the gene, had no effect on transformability (Inamine

$67 \&$ Dubnau, 1995). Although this conclusion is consistent with the absence of comEB in

68 some transformable bacteria that encode ComEA and ComEC, it remained possible that

69 the major portion of ComEA still encoded by $\operatorname{comEB} B^{484}$ was sufficient for normal

70 transformability. A recent report that ComEB plays an important role in transformation

71 (Burghard-Schrod et al., 2020) suggests that this may indeed be the case and caused us to

72 re-examine the role of this protein.

73 We show here that the ComEB protein is completely dispensable for transformation.

74 We also show that the $c o m E B$ and $\operatorname{comEC}$ open reading frames are translationally

75 coupled, explaining why deletion of $\operatorname{com} E B$ causes a loss of transformability. Finally, we

76 speculate as to the reason for coupling and for why ComEB, a dCMP deaminase

77 (Burghard-Schrod et al., 2020, Oehlenschlaeger et al., 2015), is expressed by cells that

78 are competent for transformation. 


\section{Results}

81 In B. subtilis, comEB terminates in a pair of UGA stop codons that are immediately

82 followed by the start of $\operatorname{comEC}$ (UGA UGA $A U G$ ), where the stop codons of $\operatorname{com} E B$ are

83 in boldface and the start of $\operatorname{comEC}$ is italicized (Fig. 1A). This arrangement suggests that

84 a ribosomal binding site (RBS) for comEC may be embedded in the coding sequence of

$85 \operatorname{com} E B$ and even that the two genes may be translationally coupled, so that the translation

86 of the $c o m E B$ coding sequence is required for translation of $c o m E C$ as suggested

87 previously (Inamine \& Dubnau, 1995). The transformation deficient $\triangle$ comEB::tet

88 construct used by Burghard-Schrod et al (2020) removed the entire comEB reading frame

89 including any sequences embedded in $c o m E B$ that might contain an RBS for comEC

90 translation. To explore the role of $\operatorname{com} E B$ we utilized a number of constructs, all

91 replacing the native locus in single copy, under normal competence control (Fig. 2).

92 Previous work has shown that the $\operatorname{com} E$ operon is transcribed from a promoter upstream

93 of $\operatorname{com} E A$, with a likely additional minor promoter located between $c o m E A$ and $c o m E B$

94 (Hahn et al., 1993) (Fig. 2). Thus, the deletion of sequences within comEB is a priori

95 unlikely to interfere with the transcription of either comEA or comEC.

96 The $\triangle$ comEB::ery construct (Fig. 2) replaces nearly the entire coding sequence of

$97 \operatorname{com} E B$ with an erythromycin resistance cassette, without perturbing the coding

98 sequences of $c o m E A$ and $\operatorname{comEC}$ (Koo et al., 2017). The construct retains the start codon

99 of $c o m E B$ and leaves 21 base pairs from the end of the $c o m E B$ open reading frame.

100 Although ery is oriented in the same direction as $\operatorname{comEB}$, a stop codon is present at the

101 end of the cassette, which will cause a translating ribosome to detach before reaching the

102 final 21 residues of $c o m E B$. In several experiments with this construct, a low level of

103 transformation was measured, about 40-fold lower than that of the wild-type strain, but

104 still well above the transformation frequency of a $\triangle$ comEC mutant, which is completely

105 deficient (Table 1). This result confirms the finding of Burghard-Schrod et al (2020),

106 which led to the conclusion that the ComEB protein is needed for transformation,

107 admitting two possible interpretations. Either ComEB protein is needed for

108 transformation or the deletion has removed sequences that are essential for the expression

109 of $\operatorname{com} E C$. Note that in the $\triangle \operatorname{com} E B$ ::ery construct, the promoter associated with the 
110 resistance cassette is expected to drive downstream genes (Koo et al., 2017), so that the

111 cassette should not be transcriptionally polar on comEC. To test whether the

$112 \triangle \operatorname{AcomEB}:$ :ery mutation reduced the translation of $c o m E C$, as suggested by the contiguity

113 of their coding sequences, we used Western blotting with an antiserum against a twin-

114 strep tag (TST) fused to the C-terminus of ComEC. Because ComEC is weakly

115 expressed, we used a host carrying a Pmtl-(comK comS) construct, to increase expression

116 of competence proteins (Rahmer et al., 2015). Fig. 3 shows that the $\triangle$ comEB::ery strain

117 produced no detectable ComEC, consistent with the idea that removal of $\operatorname{comEB}$

118 sequences prevents the full expression of comEC. Some undetected ComEC must be

119 present because the transformation frequency is not zero (Table 1).

120 To further between the two interpretations described above, we used the cre-

121 expressing plasmid pDR244 (Koo et al., 2017), to generate the $c o m E B^{\text {Lloopout }}$ construct

122 that removes the ery cassette together with its stop codon (Fig. 2). The resulting strain

123 carries the start codon of $\operatorname{comEB}$, a 150 base pair scar from excision of the cassette and

124 the last 21 base pairs of $\operatorname{com} E B$, which are placed in-frame with the $\operatorname{com} E B$ start codon to

125 produce a theoretical protein of 58 residues. This strain restored transformation to a level

126 an order of magnitude higher than $\triangle \operatorname{comEB}$ ::ery, although about 4-fold lower than the

127 wild-type strain (Table 1). As a further demonstration that ComEB is not needed for

128 transformation, we constructed $c o m E B^{\triangle 486}$, an in-frame deletion construct that leaves the

129 first ten codons of $c o m E B$ and 54 residues at the downstream end of the gene, thus

130 encoding an in-frame theoretical peptide of 28 residues in place of the 189 residue native

131 ComEB (Fig. 2). As shown in Table 1, the comEB ${ }^{4486}$ strain, like comEB $B^{4 l o o p o u t}$, is

132 transformable, and in fact consistently exhibits a higher transformation frequency than

133 the wild type strain. The phenotypes of the $c o m E B^{\Delta l o o p o u t}$ and $c o m E B^{\triangle 486}$ strains, together

134 with the previous report that $c o m E B^{484}$ is fully transformable (Inamine \& Dubnau, 1995),

135 show conclusively that ComEB is not needed for transformation.

136 As noted above, the $\triangle$ comEB::ery construct inserts the ery cassette with its stop

137 codon, so that the final 21 base pairs at the downstream end of $c o m E B$ should not be

138 translated. In contrast, in the $c o m E B^{\triangle 84}, \mathrm{comEB}^{\triangle 486}$ and $c o m E B^{4 l o o p o u t}$ constructs, all of

139 which retain higher transformability than $\triangle$ comEB::ery, these final 7 codons are

140 translated beginning from the start of the comEB open reading frame. Thus, it appeared 
141 possible that translation through a sequence embedded near the end of $\operatorname{com} E B$ was

142 needed to engage an RBS, which cannot work efficiently on its own. To directly test the

143 need for translation of $c o m E B$, we inserted a stop codon (TAA) in $c o m E B$, substituting

144 for the native GAA at codon 72 . This strain $\left(\operatorname{comEB} B^{S T O P}\right)$ had a transformation frequency

145 100-fold lower than the wild-type strain (Table 1). Thus, in two constructs (AcomEB::ery

146 and $c o m E B^{S T O P}$ ), when translation of $c o m E B$ was interrupted upstream of the final 7

147 codons, transformation was deficient, showing that translation through these codons is

148 required for the translation of $\operatorname{comEC}$.

149 It has been reported that in the absence of ComEB, the essential transformation

150 protein ComGA does not localize near the poles of competent cells (Burghard-Schrod et

151 al., 2020) where it normally tends to reside (Hahn et al., 2005, Kidane \& Graumann,

152 2005). This would suggest a role for ComEB aside from its lack of an essential role in

153 transformation per se. To re-examine this issue, we expressed a functional fusion of

154 green fluorescent protein (GFP) to the C-terminus of ComGA from its native promoter,

155 placed at the ectopic amyE locus (Hahn et al., 2005). Fig. 4 shows that in our hands, the

156 polar and near-polar localizations of ComGA-GFP are not noticeably affected by the

157 absence of ComEB. We have no explanation for the discrepancy between our results and

158 the imaging data of Burghard-Schrod et al (2020).

160 Discussion

162 A significant finding of this study is that the ComEB protein plays no detectable role 163 in transformation. However, some interruptions of the comEB coding sequence markedly

164 reduce the transformation frequency because they interfere with the synthesis of the

165 essential ComEC protein. Promoter mapping previously revealed that a major promoter

166 drives transcription of the entire comE operon and a possible minor promoter lies

167 between comEA and comEB (Hahn et al., 1993), indicating that deletion of comEB should

168 not interfere with the transcription of comEC by removing a promoter. The

169 transformation deficiency of the $c O m E B^{S T O P}$ strain further argues that the polarity effect is

170 not exerted on the level of transcription. 
171 Based on our results, two features of $\operatorname{comEB}$ are likely to be needed for the

172 production of ComEC; translation of the reading frame and the presence of a sequence

173 within the final 21 bases of $\operatorname{comEB}$ that acts as a suboptimal or inaccessible RBS for

174 comEC. In fact, a sequence (ACGAGCT) is present within these final few residues of

$175 \operatorname{com} E B$, containing 5 out of 7 bases complementary to a sequence near the 3 ' terminus of

176 B. subtilis 16s rRNA (Figs. 1 and S1). Perfect complementarity (AGGAGGT), defines

177 the canonical Shine-Dalgarno motif for B. subtilis (Shine \& Dalgarno, 1975, Abolbaghaei

178 et al., 2017). The positionw of this possible comEB Shine-Dalgarno motif with respect to

179 the comEC start codon ( $\mathrm{D}_{\text {toStart }}$ as defined by Prabhakaran et al. (2015)), is 22 residues, a

180 few more than the typical 18-19 residues but within the permissible range. This sequence

181 (Fig. 5), which constitutes a suboptimal RBS, is present in the three constructs that

182 exhibit normal or near normal transformation (comEB ${ }^{484}, \operatorname{comEB}^{4486}$ and comEB ${ }^{4 l o o p o u t}$ ).

183 Although the deficient phenotypes of the $c O m E B^{S T O P}$ and $\triangle c o m E B$ ::ery strains show

184 that translation of $c o m E B$ is important for the expression of $c o m E C$, the fact that they are

185 significantly more transformable than a comEC deletion strain (Table 1), argues that

186 ribosomes can probably load inefficiently without upstream translation at an RBS within

$187 \operatorname{comEB}$, probably the ACGAGCT sequence noted above. In fact the $\triangle$ comEB::tet

188 construct used by Burghard-Schrod et al (2020) removed this putative RBS and retained

189 no residual transformation, in support of this idea. We have consistently observed that the

$190 \operatorname{com} E B^{4486}$ strain exhibits a higher transformation frequency than the wild type strain,

191 whereas that of the $c o m E B^{\text {Aloopout }}$ strain is reduced several fold (Table 1). These variations

192 are consistent with the finding that translational coupling is affected by local sequence in

193 subtle and complex ways (Levin-Karp et al., 2013, Buchan \& Stansfield, 2007).

194 A translating ribosome will terminate translation from the $c o m E B$ transcript at the

195 sequence UGA UGA $A U G$. After release of the nascent ComEB protein, a ribosome may

196 be favorably positioned to engage the RBS centered at the suboptimal ACGAGCT motif

197 to initiate the translation of comEC either by direct interaction or because the local

198 concentration of ribosomes is elevated following their release. It is also possible that

199 ribosomes unfold an RNA structure during upstream translation, exposing sequences for

200 comEC translation (Rex et al., 1994, Spanjaard \& van Duin, 1989, Huber et al., 2019,

201 Saito et al., 2020). In fact, analysis of the downstream sequence of the comEB open 
202 reading frame using RNAfold (Lorenz et al., 2011), suggests a structure, with a

203 calculated free energy of $-10 \mathrm{kcal} / \mathrm{mol}$ and base pairing that would sequester the putative

204 SD motif and the comEC start codon (Fig. 5). Of course, these possibilities are not

205 mutually exclusive; both the non-canonical sequence of the RBS and the presence of the

206 stem-loop may be overcome as a result of $c o m E B$ translation. Whatever the precise

207 mechanism, translational coupling of $\operatorname{comEB}$ and $c o m E C$ renders the production of

208 ComEC, and thus transformability, largely dependent on translation of comEB.

209 Translational coupling may serve to fine-tune the synthesis of ComEC independently

210 of the DNA receptor ComEA, which may be needed in relatively large amounts to ensure

211 the uptake of DNA. Examination of the comE operon in other bacteria suggests that

212 coupling of comEB and comEC is widespread. Lactobacillus plantarum and B. anthracis

213 are similar to $B$. subtilis as their comEB and comEC reading frames are nearly contiguous

214 (Fig. 1), and so are Leuconstoc mesenteroides, L. lactis and B. licheniformis (not shown).

215 Other firmicutes, e.g. L. amylovorus and S. pneumoniae (Fig. 1), as well as B. pumilus, $S$.

216 mutans, Geobacillus thermoleovorans and the Gram negative Thermus thermophilus (not

217 shown) have actual overlaps between the comEB and comEC coding sequences.

218 Staphylococcus aureus has a well-spaced arrangement of the genes, but with an

219 apparently weak Shine-Dalgarno sequence between comEB and comEC. It seems that a

220 variety of mechanisms have evolved to down-regulate the synthesis of ComEC relative to 221 that of ComEA.

222 Interestingly, the $B$. subtilis comFC gene is preceded by $c o m F B$ that encodes a

223 protein of unknown function dispensable for transformation (Sysoeva et al., 2015). As

224 pointed out previously (Sysoeva et al., 2015), comFB and comFC overlap, suggesting

225 that they are also translationally coupled and this arrangement may serve to adjust the

226 expression of ComFC with respect to ComFA, both of which are essential for

227 transformation. Also, in the com $G$ operon, which encodes the transformation pilus, the

$228 \operatorname{com} G C, \operatorname{com} G D, \operatorname{com} G F$ and com $G G$ genes successively overlap, as noted previously

229 (Albano et al., 1989). Thus in B. subtilis, all of the major operons required for

230 transformation ( $\mathrm{com} G, \mathrm{comF}$ and $\operatorname{com} E$ ), exhibit the potential for translational coupling

231 that may serve to fine-tune expression stoichiometries for proteins that work in supra- 
232 molecular complexes, partially detaching the translation of downstream genes from strict 233 transcriptional control.

234 These data establish the dispensability of ComEB for transformation, but beg the 235 question of a possible secondary role for ComEB in the context of competence; why do 236 many firmicutes encode ComEB in the ComE operon, placing the transcription of $c o m E B$ 237 under competence control? It was suggested that ComEB aids in the proper localization 238 of ComGA in the competent cell, but our results do not support that conclusion. Perhaps 239 ComEB, which is a dCMP deaminase (Burghard-Schrod et al., 2020, Oehlenschlaeger et $240 a l ., 2015)$, plays a role in scavenging pyrimidines, including those released by

241 degradation of the non-transforming strand during DNA transport. In fact, the products of 242 this degradation include 5' deoxyribomononucleotides, released into the medium

243 (Dubnau \& Cirigliano, 1972). In this regard it is interesting that ComFC, a conserved 244 protein that is required for DNA uptake, contains a domain that resembles a type 1 245 phosphoribosyl transferase (L. Celma, S. Marsin, P. Radicella and S. Quevillon-Cheruel, 246 personal communication), another enzyme that plays a role in nucleic acid base salvage. 247 Also, transcriptional profiling suggests that the genes encoding xanthine phosphoribosyl 248 transferase and a xanthine transporter, $p b u X$ and $x p t$ respectively, are induced by ComK, 249 and thus expressed in the competent state (Berka et al., 2002). These various scavenging 250 enzymes may increase fitness in the context of competence, particularly under conditions 251 of nutritional scarcity, apart from any direct role they may or may not play in DNA 252 transport.

253 These speculations are relevant to the recurring debate over the role(s) of 254 transformation; is it for food or for genetic information or for both, as suggested by

255 Redfield (1993)? Perhaps in at least some bacteria, an enhanced ability to use the non256 transforming donor DNA and the recipient DNA displaced by integration for nutrition, 257 accompanies the induction of competence for the acquisition of genetic information, a 258 parsimonious use of resources to enhance fitness. 
262 Bacteria, growth and transformation. All of the strains (Table 2) described in this

263 report are derivatives of $B$. subtilis 168 and are isogenic with IS75 (his leu met). For

264 growth purposes other than transformation, the bacteria were grown in LB medium

265 (Kearns \& Losick, 2005). Growth to competence was by the "two-step" procedure as

266 described in Dubnau and Davidoff-Abelson (1971) except that fresh, unfrozen cells were

267 used for transformation. After growth to competence, $1 \mathrm{ml}$ of culture was incubated with

$2681 \mu \mathrm{g}$ of chromosomal DNA from BD170 (thr trp) for 30 minutes at $37^{\circ} \mathrm{C}$. Transformants

269 were selected on glucose minimal medium agar lacking leucine for selection of $\mathrm{Leu}^{+}$

270 prototrophy. Transformation frequencies were calculated as the number of prototrophic

271 transformants $\mathrm{ml}^{-1}$ divided by the number of colony forming units $\mathrm{ml}^{-1}$.

272 Introduction of markerless constructs to the $\boldsymbol{B}$. subtilis chromosome. Mutant

273 genes of interest were cloned into pMiniMAD2, a gift from Dan Kearns (Indiana

274 University). After transformation with selection for erythromycin $(5 \mu \mathrm{g} / \mathrm{ml})$, markerless

275 mutants were isolated essentially as described by Patrick and Kearns (2008). In all cases

276 the mutant constructs were confirmed by sequencing.

277 Mutant constructs. The primers used for the construction of mutant plasmids are

278 described in Table 3. All constructs were verified by sequencing.

279 comEB $\boldsymbol{B B}^{\text {4loopout }}$ (BD8923) was constructed from BD8899 (Table 2) by looping out the

280 erythromycin resistance cassette using plasmid pDR244, as described by Koo et al (2017).

$281 \quad \operatorname{com}_{\boldsymbol{E}} \boldsymbol{B}^{\mathbf{4 8 6}}$ (BD8902) was obtained by PCR amplification of the $1 \mathrm{~kb}$ regions upstream

282 and downstream of the 486 comEB reidues to delete by using respectively the primer pairs

283 up-EBfor/up-EBrev and EB-downfor/EB-downrev. The two resulting fragments were

284 cloned using the NEBuilder HiFi DNA assembly kit into pMiniMad2 plasmid previously

285 digested using the restriction sites BamHI and EcoRI. The resulting plasmid pED2386 was

286 moved into the chromosome of $B$. subtilis at the native locus as described previously

287 (Mukherjee et al., 2013).

288 comEB $^{\text {STOP }}$ (BD8915) was constructed by PCR amplification of the $1 \mathrm{~kb}$ regions

289 upstream and downstream of the $\operatorname{com} E B$ glutamate amino acid at position 72 by using the

290 primer pair EB 2kb pMM-for and EB 2kb MM-rev. The resulting fragment was cloned

291 using the NEBuilder HiFi DNA assembly kit (NEW ENGLAND BioLabs) into the

292 pMiniMAD2 plasmid previously digested using the restriction sites BamHI and EcoRI. 
293 Mutagenesis was carried out using the Phusion site directed mutagenesis kit (Thermo

294 Fisher) and primer pair EB-stop cod mod-for and EB stop-cod mod-rev. The resulting

295 plasmid pED2392 was moved into the chromosome of B. subtilis at the native locus as

296 above.

297 ComEC-TST fusion. The fusion construct comEC-Twin strep tag (plasmid pED2385) was

298 generated from a synthesized fragment purchased from Genscript USA that contained two

299 strep tag II sequences, separated by a linker and flanked with BamHI and SacI restriction

300 sites. A 998 bp DNA fragment carrying the $\mathrm{C}$ terminus of comEC was cloned into the

301 pUC19 plasmid using the restriction sites SalI and BamHI. The synthetic twin strep tag

302 fragment (93 bp) was added to the comEC gene in the resulting plasmid with the restriction

303 sites BamHI and SacI. The entire fragment carrying the comEC-twin strep tag fusion

304 construct was then inserted into the SalI and SacI sites of pUC-CM (pED2384) previously

305 digested using the same restriction sites. The resulting plasmid pED2389, was then

306 transformed into BD8928 with selection for chloramphenicol-resistance by Campbell-like

307 transformation, placing the fusion construct under control of the native comEC promoter

308 to produce BD8931. The AcomEB::kan (BD8791) construct was in turn introduced by

309 transformation to form BD8953.

310 Microscopy. After growth to the competent state, $1 \mu$ of culture was deposited on an

311 agarose pad and imaged by phase microscopy as well as for GFP fluorescence, using a

312 Nikon Ti microscope. The images in Fig. 3 were collected using an Orca Flash 4.0

313 camera (Hamamatsu) and processed identically using Elements software (Nikon) and

314 Photoshop (Adobe).

315 Western blotting. We detected ComEC using an antiserum (IBA Lifesciences)

316 against a C-terminal twin-strep tag fusion to ComEC in the Pmtl-(comK comS)

317 background (BD8931) and in an isogenic comEB::ery strain (BD8953). Membrane

318 fractions were isolated as described in Draskovic et al (2005), except that the membrane

319 fractions were simply pelleted by centrifugation at 100,000 x g and not purified further.

320 In addition to the isogenic comEB ${ }^{+}$strain, a Pmtl-(comK comS) strain with no tag

321 (BD8928) was included to confirm the identification of the ComEC-TST signal. The

322 strains were grown in LB-broth to mid-log and induced by the addition of mannitol (1\%).

323 After 90 minutes the cells were harvested for Western blotting. 


\section{Acknowledgements}

326 This work was supported by NIH grant R01GM057720. We thank Mathew Neiditch and

327 the members of the Dubnau lab for helpful comments and discussion. We thank Daniel

328 Ziegler at the Bacillus Genetic Stock Center and Jörg Stulke for generously providing

329 strains. The authors have no conflicts of interest to declare that are relevant to the content

330 of this article.

Author contributions

334 M. D. S. and J. H. performed all of the experimental work in this work. D. D. supervised 335 the work and all the authors contributed to planning experiments, analyzing data and to

336 writing the paper.

\section{References} Would Affect SD Sequences in Escherichia coli and Bacillus subtilis. G3 (Bethesda) 7: 1607-1615.

Albano, M., R. Breitling \& D. A. Dubnau, (1989) Nucleotide sequence and genetic organization of the Bacillus subtilis comG operon. J Bacteriol 171: 5386-5404.

Berka, R. M., J. Hahn, M. Albano, I. Draskovic, M. Persuh, X. Cui, A. Sloma, W. Widner \& D. Dubnau, (2002) Microarray analysis of the Bacillus subtilis K-state: genome-wide expression changes dependent on ComK. Mol Microbiol 43: 1331-1345.

Blokesch, M., (2016) Natural competence for transformation. Curr Biol 26: R1126R1130.

Buchan, J. R. \& I. Stansfield, (2007) Halting a cellular production line: responses to ribosomal pausing during translation. Biol Cell 99: 475-487.

Burghard-Schrod, M., S. Altenburger \& P. L. Graumann, (2020) The Bacillus subtilis dCMP deaminase ComEB acts as a dynamic polar localization factor for ComGA within the competence machinery. Mol Microbiol 113: 906-922.

Draskovic, I. \& D. Dubnau, (2005) Biogenesis of a putative channel protein, ComEC, required for DNA uptake: membrane topology, oligomerization and formation of disulphide bonds. Mol Microbiol 55: 881-896. 
Dubnau, D. \& M. Blokesch, (2019) Mechanisms of DNA Uptake by Naturally Competent Bacteria. Annu Rev Genet 53: 217-237.

Dubnau, D. \& C. Cirigliano, (1972) Fate of transforming DNA following uptake by competent Bacillus subtilis. III. Formation and properties of products isolated from transformed cells which are derived entirely from donor DNA.J Mol Biol 64: 9-29.

Dubnau, D. \& R. Davidoff-Abelson, (1971) Fate of transforming DNA following uptake by competent Bacillus subtilis. I. Formation and properties of the donor-recipient complex. J Mol Biol 56: 209-221.

Gangel, H., C. Hepp, S. Muller, E. R. Oldewurtel, F. E. Aas, M. Koomey \& B. Maier, (2014) Concerted spatio-temporal dynamics of imported DNA and ComE DNA uptake protein during gonococcal transformation. PLoS Pathog 10: e1004043.

Hahn, J., G. Inamine, Y. Kozlov \& D. Dubnau, (1993) Characterization of comE, a late competence operon of Bacillus subtilis required for the binding and uptake of transforming DNA. Mol Microbiol 10: 99-111.

Hahn, J., B. Maier, B. J. Haijema, M. Sheetz \& D. Dubnau, (2005) Transformation proteins and DNA uptake localize to the cell poles in Bacillus subtilis. Cell 122: 59-71.

Huber, M., G. Faure, S. Laass, E. Kolbe, K. Seitz, C. Wehrheim, Y. I. Wolf, E. V. Koonin \& J. Soppa, (2019) Translational coupling via termination-reinitiation in archaea and bacteria. Nat Commun 10: 4006.

Inamine, G. S. \& D. Dubnau, (1995) ComEA, a Bacillus subtilis integral membrane protein required for genetic transformation, is needed for both DNA binding and transport. J Bacteriol 177: 3045-3051.

Kearns, D. B. \& R. Losick, (2005) Cell population heterogeneity during growth of Bacillus subtilis. Genes Dev 19: 3083-3094.

Kidane, D. \& P. L. Graumann, (2005) Intracellular protein and DNA dynamics in competent Bacillus subtilis cells. Cell 122: 73-84.

Koo, B. M., G. Kritikos, J. D. Farelli, H. Todor, K. Tong, H. Kimsey, I. Wapinski, M. Galardini, A. Cabal, J. M. Peters, A. B. Hachmann, D. Z. Rudner, K. N. Allen, A. Typas \& C. A. Gross, (2017) Construction and Analysis of Two Genome-Scale Deletion Libraries for Bacillus subtilis. Cell Syst 4: 291-305 e297.

Levin-Karp, A., U. Barenholz, T. Bareia, M. Dayagi, L. Zelcbuch, N. Antonovsky, E. Noor \& R. Milo, (2013) Quantifying translational coupling in E. coli synthetic operons using RBS modulation and fluorescent reporters. ACS Synth Biol 2: 327-336.

Lorenz, R., S. H. Bernhart, C. Honer Zu Siederdissen, H. Tafer, C. Flamm, P. F. Stadler \& I. L. Hofacker, (2011) ViennaRNA Package 2.0. Algorithms Mol Biol 6: 26.

Maier, B., (2020) Competence and Transformation in Bacillus subtilis. Curr Issues Mol Biol 37: 57-76.

Matthey, N. \& M. Blokesch, (2016) The DNA-Uptake Process of Naturally Competent Vibrio cholerae. Trends Microbiol 24: 98-110.

Mukherjee, S., P. Babitzke \& D. B. Kearns, (2013) FliW and FliS function independently to control cytoplasmic flagellin levels in Bacillus subtilis. J Bacteriol 195: 297-306. 
Oehlenschlaeger, C. B., M. N. Lovgreen, E. Reinauer, E. Lehtinen, M. L. Pind, P. Harris, J. Martinussen \& M. Willemoes, (2015) Bacillus halodurans Strain C125 Encodes and Synthesizes Enzymes from Both Known Pathways To Form dUMP Directly from Cytosine Deoxyribonucleotides. Appl Environ Microbiol 81: 3395-3404.

Patrick, J. E. \& D. B. Kearns, (2008) MinJ (YvjD) is a topological determinant of cell division in Bacillus subtilis. Mol Microbiol 70: 1166-1179.

Prabhakaran, R., S. Chithambaram \& X. Xia, (2015) Escherichia coli and Staphylococcus phages: effect of translation initiation efficiency on differential codon adaptation mediated by virulent and temperate lifestyles. $J$ Gen Virol 96: 1169-1179.

Provvedi, R. \& D. Dubnau, (1999) ComEA is a DNA receptor for transformation of competent Bacillus subtilis. Mol Microbiol 31: 271-280.

Rahmer, R., K. Morabbi Heravi \& J. Altenbuchner, (2015) Construction of a SuperCompetent Bacillus subtilis 168 Using the P mtlA -comKS Inducible Cassette. Frontiers in microbiology 6: 1431.

Redfield, R. J., (1993) Genes for breakfast: the have-your-cake-and-eat-it-too of bacterial transformation. J Hered 84: 400-404.

Rex, G., B. Surin, G. Besse, B. Schneppe \& J. E. McCarthy, (1994) The mechanism of translational coupling in Escherichia coli. Higher order structure in the atpHA mRNA acts as a conformational switch regulating the access of de novo initiating ribosomes. J Biol Chem 269: 18118-18127.

Saito, K., R. Green \& A. R. Buskirk, (2020) Ribosome recycling is not critical for translational coupling in Escherichia coli. Elife $\mathbf{9}$.

Schwarzenlander, C., W. Haase \& B. Averhoff, (2009) The role of single subunits of the DNA transport machinery of Thermus thermophilus HB27 in DNA binding and transport. Environ Microbiol 11: 801-808.

Shine, J. \& L. Dalgarno, (1975) Terminal-sequence analysis of bacterial ribosomal RNA. Correlation between the 3'-terminal-polypyrimidine sequence of 16-S RNA and translational specificity of the ribosome. Eur J Biochem 57: 221-230. Spanjaard, R. A. \& J. van Duin, (1989) Translational reinitiation in the presence and absence of a Shine and Dalgarno sequence. Nucleic Acids Res 17: 5501-5507.

Sysoeva, T. A., L. B. Bane, D. Y. Xiao, B. Bose, S. S. Chilton, R. Gaudet \& B. M. Burton, (2015) Structural characterization of the late competence protein ComFB from Bacillus subtilis. Biosci Rep 35. 


\begin{tabular}{|c|c|}
\hline com $E$ genotype & $\underline{\text { Transformation frequency } \pm \mathbf{S D}^{\mathbf{a}}}$ \\
\hline Wild-type (IS75) & 1.00 \\
\hline$\triangle c o m E B:: e r y$ & $0.026 \pm 0.004$ \\
\hline comEB $B^{\text {4loopout }}$ & $0.27 \pm 0.14$ \\
\hline $\operatorname{com} E B^{4486}$ & $3.0 \pm 1.4$ \\
\hline comEB $B^{S T O P}$ & $0.0085 \pm 0.072$ \\
\hline$\triangle c o m E C:$ :ery & $<10^{-5, b}$ \\
\hline
\end{tabular}

454 aFor each determination, the number of colony forming units $\mathrm{ml}^{-1}$ of $\mathrm{Leu}^{+}$transformants

455 was divided by the total colony forming units $\mathrm{ml}^{-1}$ determined using LB agar. The values

456 for each strain were normalized to that of the wild type strain in the same experiment,

457 which exhibited an average frequency of $6.9 \times 10^{-4} \pm 2.9 \times 10^{-4}$ across all the

458 experiments. Each determination was performed at least three times and average

459 normalized values are shown together with standard deviations.

$460{ }^{\mathrm{b}}$ No transformant colonies were detected in $100 \mu \mathrm{l}$ of culture after transformation. 
Strains

466

467

\begin{tabular}{|c|c|c|}
\hline$\underline{\text { Strain }}$ & Genotypes $^{\text {a }}$ & Source \\
\hline$\overline{\text { IS75 }}$ & his leu met & Lab stock \\
\hline BD2899 & his leu met amyE::comGA-GFP (cat spc) & (Hahn et al., 2005) \\
\hline $\mathrm{BD} 8744^{\mathrm{b}}$ & his leu met $\triangle$ comEC::ery & (Koo et al., 2017) \\
\hline $\mathrm{BD} 8899^{\mathrm{b}}$ & his leu met $\triangle$ comEB::ery & (Koo et al., 2017) \\
\hline $\mathrm{BD} 8791^{\mathrm{b}}$ & his leu met $\triangle$ comEB::kan & (Koo et al., 2017) \\
\hline $\mathrm{BD} 8902^{\mathrm{c}}$ & his leu met comEB $B^{4486}$ & This report \\
\hline $\mathrm{BD} 8915^{\mathrm{c}}$ & his leu met comEB ${ }^{S T O P}$ & This report \\
\hline BD8916 & his leu met $\triangle$ comEB::ery amyE::comGA-GFP (cat spc) & This report \\
\hline $\mathrm{BD} 8923^{\mathrm{d}}$ & his leu met comEB Lloopout & This report \\
\hline $\mathrm{BD} 8928^{\mathrm{b}}$ & his leu met Pmtl-(comK comS) & $\begin{array}{l}\text { (Rahmer et al., } \\
\text { 2015) }\end{array}$ \\
\hline BD8931 & his leu met Pmtl-(comK comS) comEC-TST & This report \\
\hline BD8953 & his leu met Pmtl-(comK comS) comEC-TST $\triangle$ comEB::.kan & This report \\
\hline
\end{tabular}

${ }^{a}$ All of the comE constructs were confirmed by sequencing.

${ }^{\mathbf{b}}$ The $\triangle$ comEB::ery and $\triangle$ comEC::ery constructs were a gift from the Bacillus Genetic Stock Center (http://www.bgsc.org), described in (Koo et al., 2017) and were introduced by transformation into the IS75 background. The Pmtl-(comK comS) construct was a gift from Jörg Stulke.

${ }^{\mathrm{d}}$ BD8923 was made from BD8899 by looping out the Ery cassette using plasmid pDR244 
492

Primers

\begin{tabular}{|l|l|}
\hline Primer name & Primer sequence \\
\hline up-EB for & cctgcaggtcgactctagaggatccCGTTTTCAGAAACGAGCTTTTCG \\
\hline up-EB rev & ttttcaattcAAAGTATTGATTCCATGAAATTCGTTCC \\
\hline EB-down for & tcaatactttGAATTGAAAAAGATACATGAACAGGCG \\
\hline EB-down rev & ttgtaaaacgacggccagtgaattcGAAGAGCAGAAGGACGATGTAC \\
\hline EB 2kb pMM-for & cctgcaggtcgactctagaggatccTAAAATATGACGAAAGCGG \\
\hline EB 2kb pMM-rev & ttgtaaaacgacggccagtgaattcGTCCTGATATTGCCAAGAG \\
\hline EB-stop cod mod-for & caagaaccattcacgcttaaatgaatgcgattcttcaATGCTCTAAGTTTG \\
\hline EB-stop cod mod-rev & tgaagaatcgcattcatttaagcgtgaatggttcttgCGC \\
\hline
\end{tabular}

494

495

496 
499 Figure 1. Arrangements of comEB and comEC in various firmicutes, identified with their

500 strain numbers. In each case the last few codons of $c o m E B$ and the first few codons of

$501 \mathrm{comEC}$ are displayed together with their translated products. The comEB stop codons are

502 in boldface, the comEC start codons are in italics and the proposed Shine-Dalgarno motif

503 for B. subtilis is underlined. In (B), (C) and (D), the initial amino acid is shown in

504 parentheses, because the TTG start is nominally translated as leucine. Putative Shine-

505 Dalgarno sequences are underlined, when present.

507 Figure 2. comEB constructs referred to in the text. The top diagram shows the wild-type

508 B. subtilis comE operon. Dotted line boxes show deleted sequences, and the dashed boxes

509 show replacements of $\operatorname{com} E B$ by the ery cassette and the scar left by the $\operatorname{comEB} B^{\text {Aloopout }}$

510 construct. Arrows show the locations of the comE major and minor promoters (Hahn et

511 al., 1993). The $c o m E B^{484}, c o m E B^{\text {looput }}$ and $c o m E B^{4486}$ constructs leave sequences in-

512 frame with the start of $c o m E B$ and contain the final base pairs of $c o m E B$, including the

513 putative comEC RBS, depicted by black ellipses. The comEB ${ }^{\text {Aloopout }}$ construct was derived

514 from $\triangle$ comEB::ery by removal of the ery cassette. This event leaves behind a scar of 150

515 in-frame residues, indicated by the light gray box. The open and closed triangles show

516 introduced and naturally occurring stop codons, respectively. The reading frames and

517 deletions are not drawn to scale. All of the mutant constructs replaced the wild-type

518 sequence in single copy at the native locus, under normal competence control.

520 Fig. 3. A $\triangle$ comEB::ery strain (BD8953) produces less ComEC-TST than its wild-type 521 parent (BD8931). Cultures were grown in LB and induced by growth in the presence of

522 mannitol. Membrane fractions were isolated and Western blotting was carried out with an

523 antiserum against the TST of both the wild-type (lane 1) and the comEB mutant (lane 2)

524 strains. A Pmtl-(comK comS) strain lacking the TST fusion was included to show the

525 specificity of the antiserum (lane 3). Antiserum raised against the membrane protein

526 ComGA was used as a loading control. 
528 Fig. 4. ComGA-GFP localization in a $\triangle \operatorname{com} E B$ strain. Isogenic (A) wild-type (BD2899)

529 and (B) $\triangle$ comEB::ery (BD8899) strains carrying C-terminal fusions of GFP to ComGA

530 and transcribed by the promoter of the $\operatorname{com} G$ operon, were grown to competence and

531 imaged for fluorescence and by phase contrast. The left and right figures in each panel

532 show fluorescence and phase contrast images respectively. Arrows indicate the locations

533 of the dots on the phase contrast images. As expected, a minority of the cells exhibit dots

534 because competence is bimodally expressed.

536 Fig. 5. The final 12 residues of the comEB_reading frame, the intergenic 6 residues and

537 the first 14 residues of comEC are shown as mRNA. The proposed ACGAGCU Shine-

538 Dalgarno sequence and the comEC start codon are shown in boldface. The structure was

539 predicted using RNAfold (Lorenz et al., 2011). Also shown are the 15 most 3-terminal

540 residues of the $B$. subtilis 16s rRNA, with proposed complementary residues in boldface

541 (Abolbaghaei et al., 2017). The $\mathrm{D}_{\text {toStart }}$ distance defined by (Prabhakaran et al., 2015) is

542 determined by counting from the 3' terminus of rRNA to the start codon of the relevant

543 gene. In this case the distance is 22 residues. 
Fig. 1

B. subtilis 168

L. plantarum DSM 16365

B. anthracis A2084

L. amylovorus 3OSC

S. pneumoniae NCTC7465
...TTC ACG AGC TAC GTG TGA TGA ATG CGT AAT TCG CGC TTA...

$\begin{array}{llllllllllllllll}\mathrm{F} & \mathrm{T} & \mathrm{S} & \mathrm{Y} & \mathrm{V} & * & * & \mathrm{M} & \mathrm{R} & \mathrm{N} & \mathrm{S} & \mathrm{R} & \mathrm{L}\end{array}$

...TTA TTA GAT CGT GAC TAA TTG CGC GCA CTA TTT TTT...

$\begin{array}{llllllllllll}\mathrm{L} & \mathrm{L} & \mathrm{F} & \mathrm{R} & \mathrm{F} & * & (\mathrm{M}) & \mathrm{R} & \mathrm{A} & \mathrm{L} & \mathrm{F} & \mathrm{F}\end{array}$

A $\quad K \quad M \quad M \quad M \quad L \quad * \quad G \quad V \quad S \quad M \quad S$

...GCA AAA ATG ATG CTA TAA GGA GTG AGT ATG AG TTG CAA GGA CAA ...

(M) $\quad Q \quad G \quad Q$
...ATA GGA GAA AAG ACC TTT GCA AGT CTC AAA GAC CAA TTG GCT GTT TAG...

...TTG CAA GTC TCA AAG ACC AAT TGG CTG TTT AGA AAT GAT ... $\begin{array}{lllllllllllll}\text { (M) } & Q & V & S & K & T & N & W & L & F & R & N & D\end{array}$
$\begin{array}{ccccccccccc}E & K & L & K & D & Y & V & T & V & D & * \\ \text { GAA } & \text { AAA } & \text { CTT } & \text { AAA } & \text { GAC } & \text { TAT } & \text { GTT } & \text { ACA } & \text { GTG } & \text { GAT TAA... }\end{array}$

...ATG TTA CAG TGG ATT AAG AAT TTC TCT ATT ... 


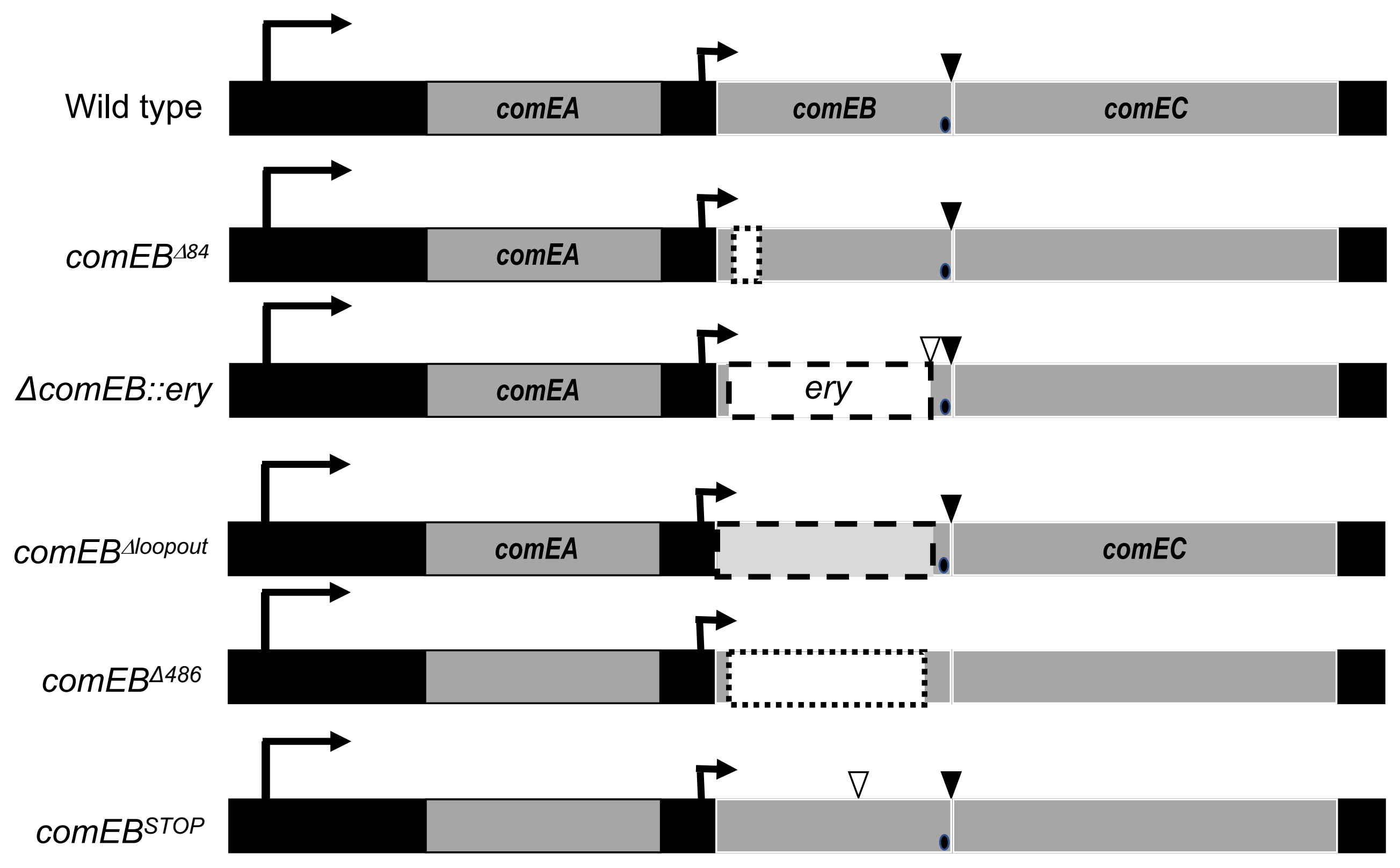



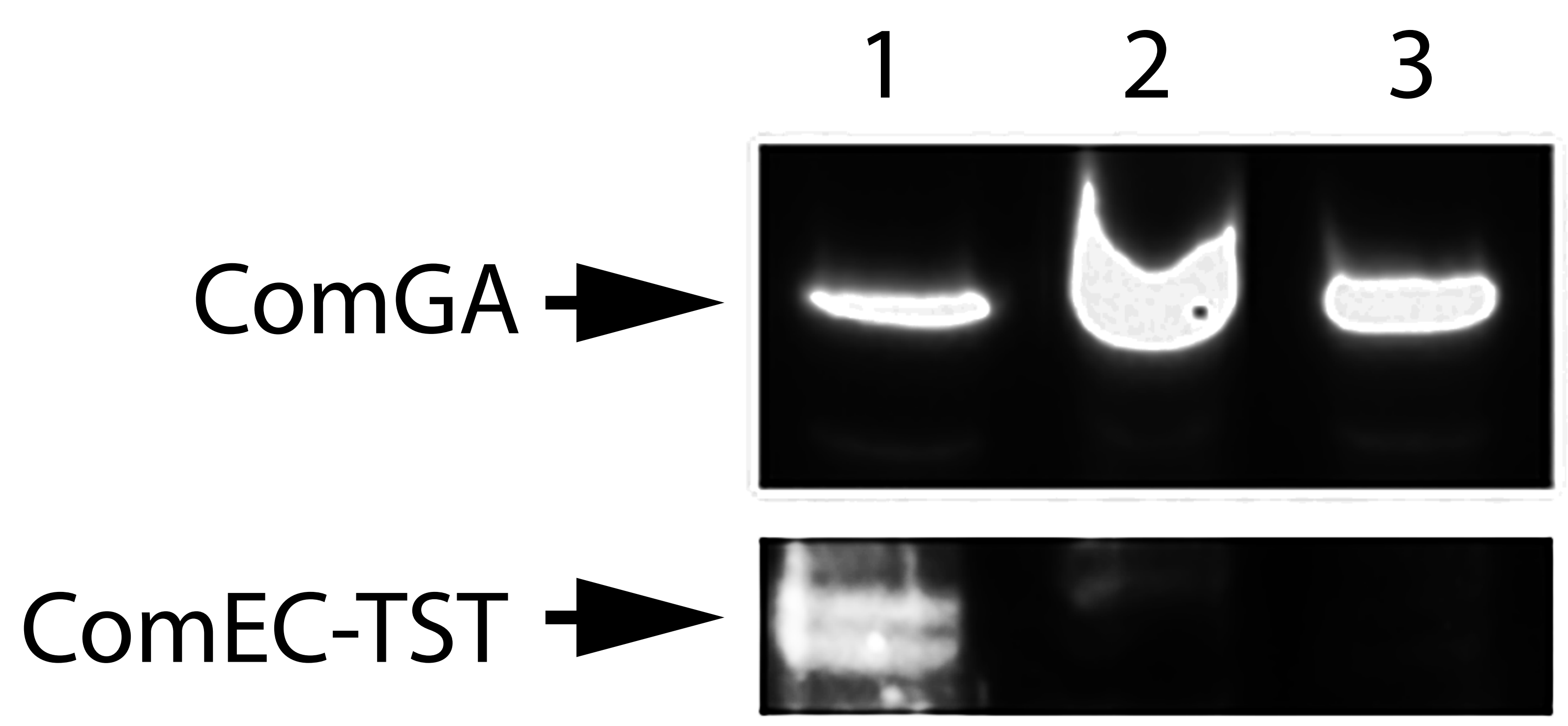


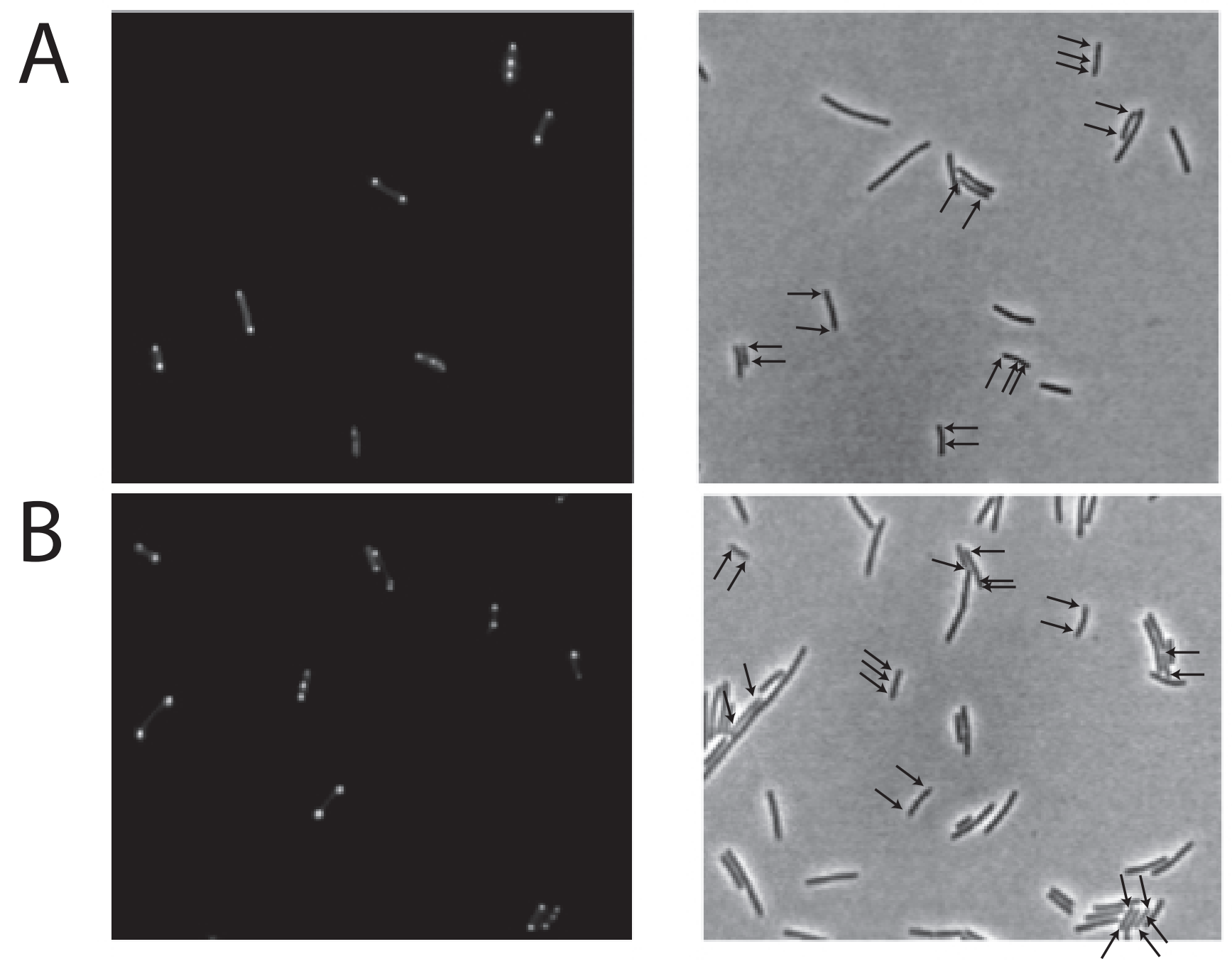




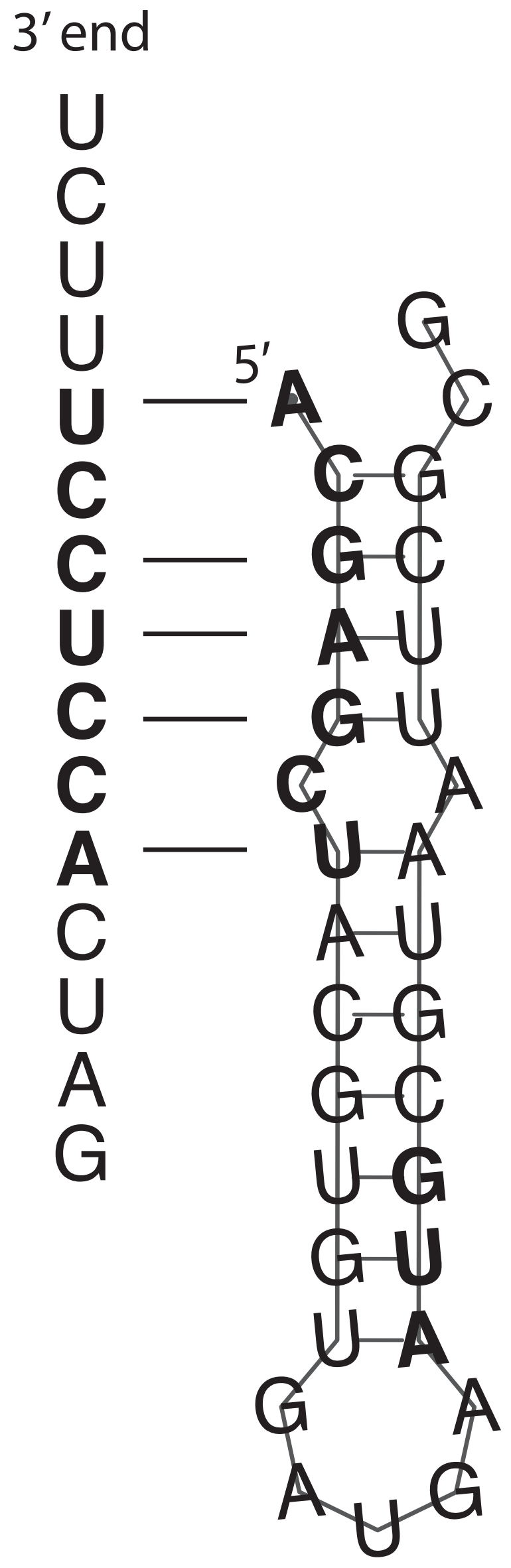

